\title{
A study on the relationship between employee mental health and agility strategic readiness: A case study of Esfahan hospitals in Iran
}

\author{
Hassan Ghodrati $^{\mathrm{a}}$ and Zahra Zargarzadeh ${ }^{\mathrm{b}^{*}}$
}

\begin{abstract}
${ }^{a}$ Accounting \& Management Department, Kashan Branch, Islamic Azad University, Kashan, Iran ${ }^{b}$ Kashan Branch, Islamic Azad University, Kashan, Iran

C H R O N I C L E

Article history:

Received December 2, 2012

Received in revised format

10 March 2013

Accepted 18 March 2013

Available online

March 192013

Keywords:

Strategic Readiness

Organizational Agility

Mental Health

Crisis Management

This study investigates whether enhancing organizational agility and mental health of staff could increase strategic readiness for crises or not. In this study, descriptive statistics is used to addition, to examine research hypotheses, correlation coefficients and descriptive statistics are implemented. Finally, to rank the variables and indicators of the research, Friedman test and for comparison of indicators and components of the research, nonparametric Kruskal-Wallis test are used. The proposed study designs a questionnaire and The questionnaire and distributes it among some nurses in obstetrics and anesthesiology department and among supervisors. Cronbach's alpha is also employed for determining the reliability in this study. The results the employees with higher levels of mental health have higher readiness to deal with potential crises, and the relationship between agility of hospitals and their strategic readiness for dealing with crises is confirmed.
\end{abstract}

\section{Introduction}

Today, manufacturing and service organizations are working in ever-changing environmental conditions, and economic, commercial, cultural, social and political conditions of environments influence organizational performance. Obviously, disasters such as war, earthquakes, floods etc. have their own influences on organizational behavior. Therefore, managers need to know that there are various environmental threats to the life of organizations, and organizations can overcome such crises only by means of strategic planning and strategic readiness (Ryvicker, 2009). According to Seifert (2007), the nursing shortage increasingly is concentrating on retaining the "aging" nurse. By growing interest in implementing this group of health care workers, there are associated questions about real and perceived chronologic limits-physiologic, cognitive, and functional-affecting older nurses.

\footnotetext{
Corresponding author. Tel: +989132761159

E-mail: z_zargarzadeh@yahoo.com (Z.Zargarzadeh)

(C) 2013 Growing Science Ltd. All rights reserved.

doi: $10.5267 /$ j.msl.2013.03.018
} 
Seifert (2007) discussed these issues in light of research associated with older perioperative nurses, aging surgeons, and other health care workers. Sznelwar et al. (2008) studied working in public health services in Brazil and investigated the relationship between different rationalities. They explained how changes implemented in Sao Paulo, Brazil could influence the organizational parameters and working activities for front-line workers.

Baba et al. (1999) discussed issues of occupational mental health among nurses in the Caribbean. They used a linear model linking role, work and social factors, stress, burnout, depression, absenteeism and turnover intention for their research. Their results indicated burnout was the sole predictor of depression, which predicted both absenteeism and turnover intention.

Lang et al. (2004) performed an investigation to detect whether the peer-reviewed literature could supports specific, minimum nurse-patient ratios for acute care hospitals and whether nurse staffing could be associated with patient, nurse employee, or hospital outcomes. The results of the survey found no support for specific, minimum nurse-patient ratios for acute care hospitals, especially in the absence of adjustments for skill and patient mix, although total nursing hours and skill mix appeared to influence some important patient outcomes.

\section{Research Hypotheses}

The main hypothesis in this research is as follows,

The employee's mental health has a positive impact on the agility and strategic readiness of hospitals in Isfahan province.

This hypothesis is divided into the following three minor hypotheses,

1. Mental health of employees has a positive impact on the hospital agility.

2. Mental health of employees has a positive impact on the strategic readiness of hospitals for responding to crisis management.

3. Agility of hospitals has a positive impact on the strategic readiness of hospitals for responding to crisis management.

\subsection{Research Methodology}

The methods used in this study include: applied method concerning the objective of the research (the reasons for conducting the research), and descriptive-analytic approach for inference, and finally survey method for design of the research. Statistical community includes all 30,000 employees of Esfahan Hospitals and we randomly selected 320 people from 80 hospitals in the province. In this study, descriptive statistics has been used for presentation of demographic data of the research, and for this purpose, the demographic data is shown using frequency table. Also for studying the central indicators and dispersion of the variables of the research, descriptive statistics techniques have been used.

Inferential statistical techniques have also been used in this study and since this study sought to test the research hypotheses, the testing hypotheses and its related statistical techniques will be used. For analyzing the data, P-Test has been used and the reason for using this test at this stage is the fact that this research is a qualitative one. Also for checking and testing research hypotheses, by correlation coefficient and descriptive statistics, the status of indicators and research hypotheses have been tested. 
Since this research has been conducted in real, objective, and live (dynamic) organizations and the researcher has experienced real conditions of organizations, this study is also among field studies. Ultimately, in order to rank the variables and indicators of the research, Friedman test and for comparison of indicators and components of the research, nonparametric Kruskal-Wallis test is used and the Cronbach's alpha is employed for determining the reliability in this study. Fig. 1 shows the framework of the proposed study.

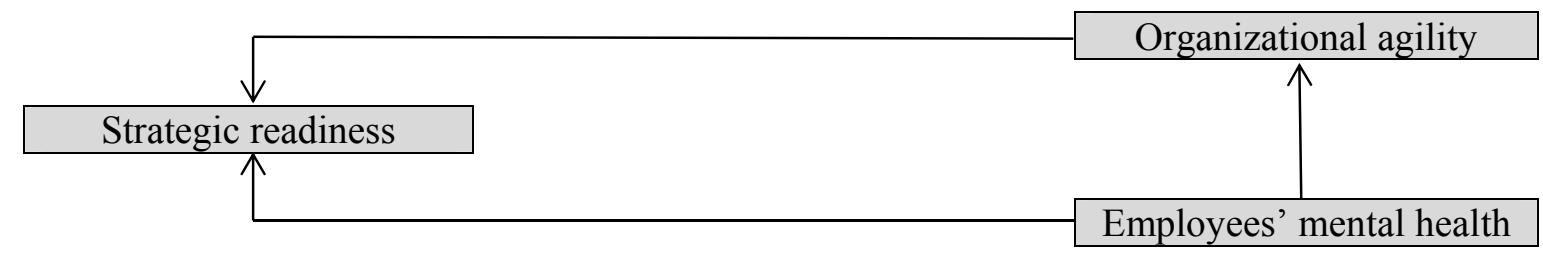

Fig. 1. The framework of the proposed study

Strategic readiness for crisis management means the level of an organization for better management before a crisis occurs. Readiness for a crisis is a strategy, because it provides a selectable model for inhibition or continuation of next organizational activities and estimates the depth of outcomes (Pennings, 1985). In this study, to assess the strategic readiness for crisis management, the measurement tool prepared by Reilly in 1987 for measuring the strategic readiness to crisis management in some US banks. The research was also been used for some hospitals in Egypt and it was validated in other countries. For measuring the strategic readiness of the concerned organizations, they studied such organizations from six aspects and the presented questions were in line with these dimensions (See Table 2).

Table 2

Strategic readiness

\begin{tabular}{|c|c|c|}
\hline Concept & Strategic readiness & \\
\hline Dimensions & $\begin{array}{ll}\text { - } & \text { Quick response capability } \\
\text { - } & \text { Timely identification of crisis } \\
\text { - } & \text { Access to crisis management resources }\end{array}$ & $\begin{array}{ll}\text { - } & \text { Internal information flow } \\
\text { - } & \text { External information flow } \\
\text { - } & \text { Crisis Planning }\end{array}$ \\
\hline
\end{tabular}

\subsubsection{Agility}

According to Zhang and Sharifi (2000), agility means the ability of each organization in sensing, understanding and predicting changes in the business environment. In fact, agility includes capabilities and competencies, which lead to survival of organization's development in business environment (with constant change and uncertainty as its main characteristics) (Khoshsima, 2002). The dimensions of agility in public sector could be explained as follows,

1- Organizational change: Recognition of citizens' requirements, improving their services, decisionmaking by public needs and implementing resources to meet customer needs.

2- Organizational leadership: Development in organizational vision, trends and strategic objectives, processing flexibility and utilization of resources based on possible needs.

3- Organizational culture and values: building a good environment to promote changes, considering urgent needs to invest in innovation and creating a sense of teamwork throughout the organization. 
4- Customer service: developing sustainable relationships among citizen to improve management strategy, aligning customer services with business processes as well as providing incentives for citizens to go towards new and cheaper communication channels.

5- E-government: developing electronic processes, implementing technology to improve administrative communications, and encouraging citizens to go for more efficient communication channels.

6- Performance management: preparing appropriate training for employees, building a comprehensive performance management system within firm, and incorporating appropriate models for assessing performance (Table 3).

Table 3

Organizational agility requirements

\begin{tabular}{|c|c|c|}
\hline Concept & Organizational agility & \\
\hline Dimensions & $\begin{array}{ll}\text { - } & \text { Organizational change } \\
\text { - } & \text { Organizational leadership } \\
\text { - } & \text { Organizational culture and values }\end{array}$ & $\begin{array}{ll}\text { - } & \text { Customer service } \\
\text { - } & \text { E-government } \\
\text { - } & \text { Performance management }\end{array}$ \\
\hline
\end{tabular}

\subsubsection{Mental Health}

According to Neeb (2005), mental health means the health of mind and indicates showing positive status and mental health, and the ways for achieving it, which plays an important role in evolution of individuals and society. In this study, to evaluate the mental health, the measurement tool developed by Goldberg (Regier et al., 1978) is implemented where the objective is to diagnose mental disorders in different centers and environments. General Health Questionnaire (GHQ) which deals with the mental status of individuals for the last month includes four domains: (1) hypochondriasis, including questions about physical symptoms, (2) anxiety and psychological distress, (3) impairment in social functioning and (4) depression.

\section{Table 4}

Organizational agility

Concept Organizational agility

\begin{tabular}{llll}
\hline \multirow{2}{*}{ Dimensions } & - & Physical symptoms & - \\
& - & Impairment in social functioning \\
\end{tabular}

\section{The results}

In this section, we present statistical results by prioritizing the main components of the research, then measure the effects of demographic factor on the research variables and finally, we test research hypotheses.

\section{Prioritization of Research Indicators}

In the present study, each component consists of several indicators and the level of influence of various indicators on the related component is different. So, we continue by ranking indicators to examine the extent of their influence on the respective components.

\subsection{Organizational Agility}

The most important indicators associated with organizational agility are summarized as follows, 
Table 5

Prioritization of the organizational agility indicators

\begin{tabular}{cllll}
\hline Row & Studied variable & Average rank & Studied variable & Average rank \\
\hline 1 & Provision of services & 4.80 & Organizational change & 3.30 \\
2 & Organizational values and culture & 3.53 & Performance management & 3.10 \\
3 & E-government & 3.51 & Organizational leadership & 2.60 \\
\hline
\end{tabular}

\subsection{Mental Health of Employees}

The most important indicators associated with mental health of employees are summarized in Table 6 as follows,

Table 6

Prioritization of the mental health of employee's indicators

\begin{tabular}{clc}
\hline Row & Studied variable & Average rank \\
\hline 1 & Impairment in social functioning scale & 3.75 \\
2 & Physical health scale & 2.80 \\
3 & Anxiety scale & 2.20 \\
4 & Depression scale & 1.20 \\
\hline
\end{tabular}

\subsection{Strategic Readiness}

The most important indicators related to strategic readiness are summarized in Table 6 as follows,

\section{Table 7}

Prioritization of the strategic readiness indicators

\begin{tabular}{cllll}
\hline Row & Studied variable & Average rank & Studied variable & Average rank \\
\hline 1 & $\begin{array}{l}\text { Access to crisis management } \\
\text { resources }\end{array}$ & 3.90 & $\begin{array}{l}\text { Timely identification of the } \\
\text { crisis }\end{array}$ & 2.80 \\
2 & Quick response capability & 3.76 & Crisis Planning & 2.50 \\
3 & Internal information flow & 3.20 & External information flow & 2.10 \\
\hline
\end{tabular}

\subsection{The Impact of Biographical Factors on the Answers}

The first part of the questionnaire is associated with demographic questions. In this section, we evaluate the impact of demographic variables on the research variables. For this reason, we used ranking analysis of variance or ANOVA. By using Kruskal-Wallis test, we also evaluated the impact of demographic factors on the responding answers.

\subsubsection{Age}

Table 11 shows that margin of error was lower than 5\%, so "age" has influenced the answers.

\section{Table 11}

The impact of age

\begin{tabular}{lccc}
\hline & Agility & Mental health of employees & Strategic readiness \\
\hline Chi-square & 253.659 & 253.350 & 253.659 \\
Degrees of freedom & 3 & 3 & 3 \\
Error & 0.000 & 0.000 & 0.000 \\
\hline
\end{tabular}




\subsubsection{Marital status}

Table 12 shows that margin of error was lower than 5\%, so "bachelors" show higher influence.

Table 12

The impact of martial effect

\begin{tabular}{lccc}
\hline & Agility & Mental health of employees & Strategic readiness \\
\hline Chi-square & 221.416 & 221.597 & 221.160 \\
Degrees of freedom & 1 & 1 & 1 \\
Error & 0.000 & 0.000 & 0.000 \\
\hline
\end{tabular}

\subsubsection{Gender}

Table 13 shows that margin of error was lower than 5\% and "female" shows higher influence.

Table 13

The impact of gender

\begin{tabular}{cccc}
\hline & Agility & Mental health of employees & Strategic readiness \\
\hline Chi-square & 197.293 & 198.554 & 196.095 \\
Degrees of freedom & 1 & 1 & 1 \\
Error & 0.000 & 0.000 & 0.000 \\
\hline
\end{tabular}

\subsubsection{Education degree}

Table 14 shows that margin of error was lower than 5\%, so "higher education degree" shows higher influence.

Table 14

Educational background

\begin{tabular}{lccc}
\hline & Agility & Mental health of employees & Strategic readiness \\
\hline Chi-square & 220.624 & 222.245 & 219.804 \\
Degrees of freedom & 2 & 2 & 2 \\
Error & 0.000 & 0.000 & 0.000 \\
\hline
\end{tabular}

\subsubsection{Work experience}

Table 15 shows that margin of error was lower than 5\%, so "more work experience" shows higher influence.

Table 15

The impact of job experience

\begin{tabular}{lccc}
\hline & Agility & Mental health of employees & Strategic readiness \\
\hline Chi-square & 250.073 & 250.447 & 249.147 \\
Degrees of freedom & 2 & 2 & 2 \\
Error & 0.000 & 0.000 & 0.000 \\
\hline
\end{tabular}

\subsubsection{Job Position}

Table16 shows that margin of error was lower than 5\%, so "higher position" shows higher influence. 
Table 16

The impact of job position

\begin{tabular}{lccc}
\hline & Agility & Mental health of employees & Strategic readiness \\
\hline Chi-square & 216.736 & 217.121 & 215.601 \\
Degrees of freedom & 4 & 4 & 4 \\
Error & 0.000 & 0.000 & 0.000 \\
\hline
\end{tabular}

\subsection{Research Hypotheses Testing}

In this section, the results of testing different hypotheses are presented.

Hypothesis No. 1: mental health of employees has a positive impact on hospital agility.

Based on the results of Table 8 , by considering the correlation coefficient, the research hypothesis is accepted. As we show, the coefficient relationship is 0.992 and there is a strong and direct relationship among these variables. A computed, the significance level is zero approximately equal to zero and it is less than 0.05 , so we accepted the $\mathrm{H}_{1}$ or relationships among variables.

Table 8

Relationship between mental health with hospital agility

\begin{tabular}{ccc}
\hline Test & Correlation coefficient & Significance level \\
\hline Spearman & 0.992 & 0.000 \\
\hline
\end{tabular}

Hypothesis No. 2: Employees' mental health has a positive impact on the strategic readiness of hospitals on responding to crisis management.

Based on the results of Table 9, by considering the correlation coefficient, the research hypothesis is accepted. The coefficient relationship is 0.984 and there is a strong and direct relationship between these variables. The significance level is less than 0.05 , so we can accept the $\mathrm{H}_{1}$ or relationship between variables hypothesis.

Table 9

Correlation between mental health and strategic readiness

\begin{tabular}{ccc}
\hline Test & Correlation coefficient & Significance level \\
\hline Spearman & 0.984 & 0.000 \\
\hline
\end{tabular}

Hypothesis No. 3: Agility of hospital has a positive impact on strategic readiness of hospital for responding to crisis management.

Finally, based on the results of Table 10, the research hypothesis is accepted based on the related correlation coefficient. The coefficient relationship is 0.995 and there is a strong and direct relationship between these variables. As we computed, the significance level is less than 0.05 , so we can accept $\mathrm{H}_{1}$.

\section{Table 10}

Correlation between strategic readiness and agility of hospital

\begin{tabular}{ccc}
\hline Test & Correlation coefficient & Significance level \\
\hline Spearman & 0.995 & 0.000 \\
\hline
\end{tabular}




\section{Conclusion}

Using various tests, including both descriptive and inferential tests including P-Tests, correlation and Friedman's test of analysis of variance, we have examined different hypotheses as follows,

\section{Mental health of employees has a positive impact on hospital agility.}

Since the null hypothesis is rejected, there should be a significant correlation between the two variables. Spearman's correlation test indicates a relationship between employees' mental health and organizational agility. The result of correlation test shows that the significance level is less than $\alpha=$ $0.05(\mathrm{P}-\mathrm{V}<\alpha=5 \%)$. Therefore, we can state that, at confidence level of $95 \%$, there is a significant relationship between mental health of employees and organizational agility. The level or intensity of correlation between mental health of employees and agility is approximately 0.992 . This suggests that per one unit of increase or improvement in mental health of employees in the studied organizations, agility will increase about 0.992 .

2. Employees' mental health has a positive impact on the strategic readiness of hospitals on responding to crisis management.

Since the null hypothesis is rejected, there should be a significant correlation between the two variables. Ultimately, Spearman's correlation test indicates that there is a relationship between mental health and strategic readiness to deal with crises. In other words, the improvement of mental health of staff directly influences the level of readiness for responding to crises. The result of Spearman's correlation test shows that the significance level is less than $\alpha=5 \%(\mathrm{P}-\mathrm{V}<\alpha=5 \%)$.

Based on the result of the performed test, it can be stated that, at confidence level of $95 \%$, the organizations with mentally healthier employees have more strategic readiness for responding to crises. The level or intensity of correlation between mental health of employees and strategic readiness is approximately 0.984 . This suggests that per one unit of increase or improvement in mental health of employees in the studied organizations, strategic readiness of these organizations for responding to crises will increase about 0.984 .

\section{3 - Agility of hospital has a positive impact on strategic readiness of hospital for responding to crisis management.}

Since the null hypothesis is rejected, there should be a significant correlation between the two variables. Pearson's correlation test indicates a relationship between agility of hospitals and their strategic readiness. The result of Person's test shows that the significance level is less than $\alpha=5 \%$ (P$\mathrm{V}<\alpha=5 \%$ ). With regard to the result of the performed test, it can be stated, at confidence level of $95 \%$, that: an organization with higher agility, strategic readiness for crisis management is higher. The level or intensity of correlation between agility of hospitals and their strategic readiness is approximately 0.995 . This suggests that per one unit of increase or improvement in agility of studied hospitals, strategic readiness of them for responding to crises will increase about 0.995 .

The results of examining the variables, indicators and the relationship between dependent and independent variables associated with hypothesis 3 indicates the existence of a relationship between agility of hospitals and their strategic readiness to deal with the crises. Theoretical discussions and review of literature also confirms such relationship. The results of the investigation conducted for hypothesis 3 and examining the relationship between dependent and independent variables associated with the this hypothesis also confirms such relationships. 
Based on the research results we recommended that:

1. Since the relationship between organizational agility and strategic readiness for responding to crisis was confirmed in the studied organizations, the following recommendations can be proposed:

- External and internal environmental factors for organizations should be carefully analyzed.

- Appropriate plans should be developed for preparing managers and staff in these organizations to deal with potential crises.

- By increasing knowledge, awareness and skills of employees and updating these factors, change should be encouraged in organizations.

- With regard to the influence of objectives and strategies in organizational agility, the organization's goals and strategies and policies should be revised in proportion to occurred environmental changes and after studying the status quo.

2. Conducted studies and surveys indicate that there is a relationship between leadership style and mental health in organizations. Therefore, it is recommended that:

- Since the job environment in hospitals are stressful and they should put up with different stress, we offer what Taylor originally recommended. In other words, suitable job description should be prepared, the ambiguities in working standards and criteria should be eliminated, the relationships between managers and employees and inter-personal relations should be strengthened, the conflicts in roles should be reduced and some actions should be taken for improving the mental health of employees in workplaces.

\section{Acknowledgment}

The authors would like to thank the anonymous referees for constructive comments on earlier version of this work.

\section{References}

Baba, V. V., Galperin, B. L., \& Lituchy, T. R. (1999). Occupational mental health: A study of workrelated depression among nurses in the Caribbean. International Journal of Nursing Studies, 36(2), 163-169.

Lang, T. A., Hodge, M., Olson, V., Romano, P. S., \& Kravitz, R. L. (2004). Nurse-patient ratios: a systematic review on the effects of nurse staffing on patient, nurse employee, and hospital outcomes. Journal of Nursing Administration, 34(7-8), 326.

Neeb, K.C. (2005). Fundamentals of Mental Health Nursing. $3^{\text {rd }}$ ed. F.A. Davis Company.

Khoshsima, G. (2002). Proposing a model for measuring agility in manufacturing organizations in the electronics industry in Iran using fuzzy logic. MS thesis, Tehran University, School of Management, Tehran.

Pennings, J.M. (1985). Organizational Strategy and Change. Jossey Bass Business and Management Series.

Regier, D. A., Goldberg, I. D., \& Taube, C. A. (1978). The de facto US mental health services system: a public health perspective. Archives of General Psychiatry, 35(6), 685.

Ryvicker, M. (2009). Preservation of self in the nursing home: Contradictory practices within two models of care. Journal of Aging Studies, 23(1), 12-23

Seifert, P.C. (2007). The "Aging" nurse: Issues, myths, and retention strategies. Perioperative Nursing Clinics, 2(4), 289-299.

Sznelwar, L.I., Silva, M.T., \& Mascia, F.L. (2008). Working in public health services in Brazil: The relationship between different rationalities. Applied Ergonomics, 39(4), 500-508 
1104

Zhang, Z., \& Sharifi, H. (2000). A methodology for achieving agility in manufacturing organisations. International Journal of Operations \& Production Management, 20(4), 496-513. 